\title{
A new self scaling PCG algorithm with dynamical retards
}

\author{
ABBAS Y. AL-BAYATI \& ASEEL MOAYAD QASIM \\ Department of mathematics \\ College Of Computer Sciences and Mathematics \\ Mosul University \\ Received \\ 04/03/2007 \\ Accepted \\ 09/05/2007

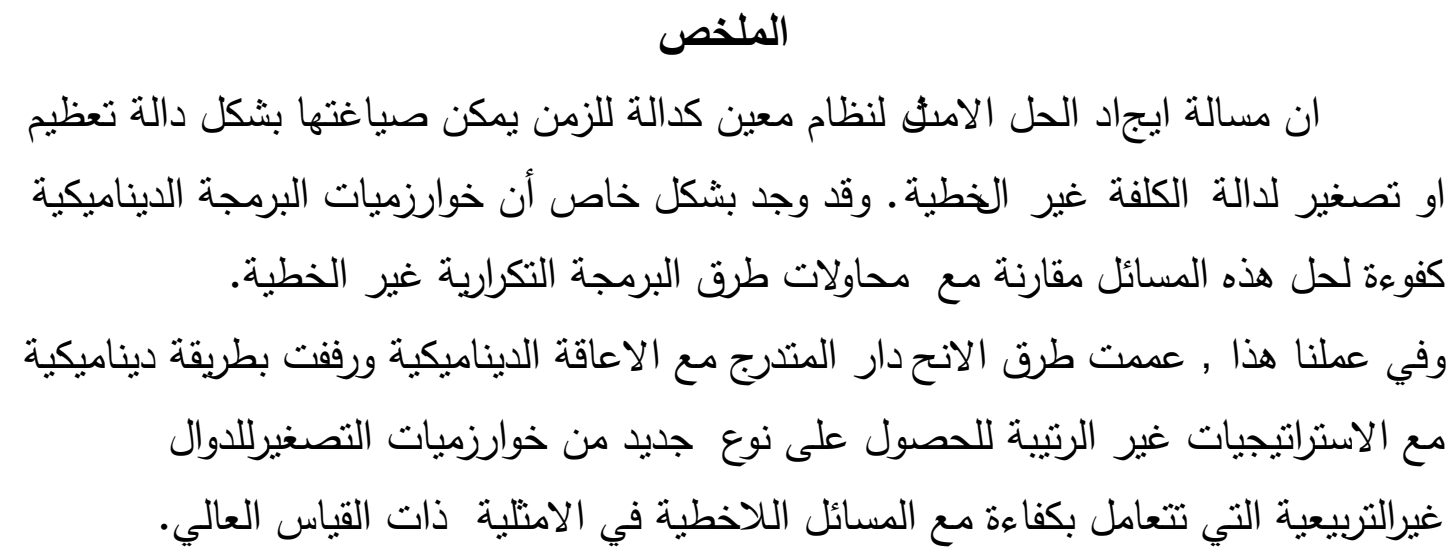

\section{Abstract}

The problem of optimizing a certain systems as a function of time may be formulated in terms of maximizing or minimizing a non-linear cost function. It was found that a Dynamic Programming (DP) algorithm was particularly efficient procedure for solving this problem compared with an alternative nonlinear programming method.

In this work, a new CG method with dynamical retards is generalized and combined in a dynamical way with non-monotone globalization strategies to obtain a new type CG-algorithm for minimizing non-quadratic functions that can deal efficiently with large scale nonlinear optimization problems.

\section{Introduction:}

Conjugate gradient (CG) algorithms have advantage over both modifiedNewton and variable metric (VM) algorithms for unconstrained optimization of not requiring a matrix store. They tend, however, to require more function evaluations to obtain the minimum than the latter algorithms and therefore their use is usually restricted to the solution of problem in a large number of dimensions, or to computers with a restricted store.

We consider the unconstrained minimization problem

$$
\min _{x \in R^{n}} f(x) \text {, }
$$


where $f: \Re^{n} \longrightarrow \Re$ is continuously differentiable and its gradient is available. We are interested in the large-scale case for which the Hessian of $f$ is either not available or requires a prohibitive amount of storage and computational cost.

The most important features of these methods are that only gradient directions are used, that the memory requirement are minimal, and they do not involve a decrease in the objective function, which allows fast local convergence.

In this paper, we extended the gradient method and introduce a new gradient method with dynamical retards to find the unique global minimizer of the quadratic function of form

$f(x)=\frac{1}{2} x^{T} G x-b^{T} x$,

where $G \in \mathfrak{R}^{n \times n}$ is large, sparse, symmetric and positive definite.

For the non-quadratic case, the method needs to be incorporated in a globalization strategy, since the method does not enforce decrease in the objective function, a non-monotone line search strategy will be used.

In particular, the non-monotone line search technique introduced by Grippo, Lampariello and lucidi [8] has proved to be very effective for large scale optimization problems. This line search essentially enforces the following condition

$f\left(X_{k+1}\right) \leq \max _{0 \leq j \leq M} f\left(X_{k-j}\right)+\gamma g_{k}^{T}\left(X_{k+1}-X_{k}\right)$,

Where $\mathrm{M}$ is a non-negative integer and $\gamma$ is a small positive number. This condition leads us to create two algorithms; the first which is called (The original) using this condition only, and the second which called (The new) using Armijo rules with BFGS updating formula and self scaling Variable Metric (VM) update for the scalar Hestense and Stiefel (HS) [9].

This paper is organized as follows: In the next section we discuss the line search procedure and then talking about Steepest Descent (SD) method and in the third section we describe a review of conjugate gradient method and in the fourth section the non-monotone gradient methods will be describe and in the fifth section we describe VM methods and the new Preconditional Conjugate Gradient (PCG) algorithm and some new theorems, while section six gives the numerical results and the conclusions, sections seven and eight contain the Appendix and the References.

\section{Line search procedure:}


One-dimensional search is the backbone of many algorithms for solving a nonlinear programming algorithms precede as follows: given a point $x_{k}$, find a direction vector $d_{k}$ and then a suitable step-size $\lambda_{k}$, yielding a new point $x_{k+1}=x_{k}+\lambda_{k} d_{k}$; the process is then repeated. Finding the step-size $\lambda_{k}$ involves solving the sub problem to minimize $f\left(x_{k}+\lambda d_{k}\right)$, which is a one-dimensional search problem in the variable $\lambda$. The minimization may be over all real $\lambda$, non-negative $\lambda$, or such that $x_{k}+\lambda d_{k}$ is feasible. Consider a function $\theta$ of one variable $\lambda$ to be minimized. One approach to minimizing $\theta$ is to set the derivative $\theta^{\prime}$ equal to 0 and then solve for $\lambda$. Note, however, that $\theta$ is usually defined implicitly in terms of a function $f$ of variables. In practice, given the vector $\mathrm{x}$ and $\mathrm{d}, \theta(\lambda)=f(\mathrm{x}+\lambda \mathrm{d})$. If $f$ is not differentiable , then $\theta$ will not be differentiable. if $f$ is differentiable, then $\theta^{\prime}(\lambda)=d^{\prime} \nabla f(x+\lambda d)=0$. Therefore, to find a point $\lambda$ with $\theta^{\prime}(\lambda)=0$, we have to solve the equation $d^{\prime} \nabla f(x+\lambda d)=0$, which is usually nonlinear in $\lambda$. Furthermore, $\lambda$ satisfying $\theta^{\prime}(\lambda)=0$ is not necessarily a minimum; it may be a local minimum, a local maximum, or even a saddle point, for these reasons, and except for some special cases, we avoid minimizing $\theta$ by letting its derivative be equal to zero. Instead, we resort to numerical techniques for minimizing the function $\theta$. In our work we use this technique to find $\lambda$ as follows:

find $\quad \mathrm{z}=\min (\mathrm{k}, \mathrm{M})$, where $\mathrm{k}$ is the count, $\mathrm{M}$ is non negative integer and for $0 \leq j \leq z$, find $\operatorname{Max} f_{(k-j)}-\gamma \cdot \lambda \cdot g_{k} g_{k}^{T}$ where $\mathrm{g}$ is the gradient of the function $f, \gamma \in(0,1), \delta \in(0,1)$. If $f\left(x_{k+1}\right)>\max f(k-j)-\lambda \cdot \gamma \cdot g_{k} \cdot g_{k}^{T}$ choose $\lambda=\delta . \lambda \quad, x_{k+1}=x_{k}+\lambda d_{k} \quad$ else $\quad \lambda_{k}=\lambda, \quad x_{k+1}=x_{k}+\lambda_{k} d_{k}$ update $\lambda$ by take $\alpha=\frac{v^{T} y}{v^{T} v}$ and $\lambda=1 / \alpha$.

\subsection{Steepest -Descent (SD):}

The Steepest Descent (SD) method is particularly useful when the dimension of the problem is very large, however, it may generate short zigzagging displacements in a neighborhood of a solution.

For simplicity, we denote $\nabla f\left(x_{k}\right)$ by $g_{k}, f\left(x_{k}\right)$ by $f_{k}$ and $f\left(x^{*}\right)$

by $f^{*}$, respectively, where $x^{*}$ denotes a local minimizer of $f$.

In the algorithmic framework of (SD) method the iterative formula $x_{k+1}=x_{k}+\lambda_{k} d_{k}, k=0,1,2$, .

Where $d_{k}$ satisfies the relation 
$g_{k}^{t} d_{k}<0$,

Which guarantees that $d_{k}$ is a descent direction of $f(\mathrm{x})$ at $x_{k}$. In order to guarantee the global convergence, it is usually required the descent condition

$g_{k}^{T} d_{k} \leq-c\left\|g_{k}\right\|^{2}$,

Where $\mathrm{c}>0$ is a constant. The angle property

$\cos \left(g_{k}^{T} d_{k}\right)=-\frac{g_{k}^{T} d_{k}}{\left\|g_{k}\right\| \cdot\left\|d_{k}\right\|} \geq \eta_{0}$

is often used in many situations, with $\eta_{0} \in(0,1]$.

Observe that, if $\left\|g_{k}\right\| \neq 0$ then $d_{k}=-g_{k}$ satisfies (5), (6) and (7) simultaneously.

There are many alternative line-search rules to choose $\lambda_{k}$ along the ray $d_{k+1}=\left\{x_{k}+\lambda_{k} d_{k} \mid \lambda>0\right\}$.namely:

(a) Minimization Rule. At each iteration, $\lambda_{k}$ is selected so that

$$
f\left(x_{k}+\lambda_{k} d_{k}\right)=\min _{\lambda>0} f\left(x_{k}+\lambda_{k} d_{k}\right)
$$

(b) Armijo Rule. Set scalars $d_{k}, \gamma, L$ and $\delta$ with $d_{k}=-\frac{g_{k}^{T} d_{k}}{L\left\|d_{k}\right\|^{2}}, \gamma \in(0,1)$, $L>0$ And $\delta \in(0,1 / 2)$.Let $\lambda_{k}$ be the largest $\lambda$ in $\left\{d_{k}, \gamma d_{k}, \gamma^{2} d_{k}, \ldots ..\right\}$ such that $f_{k}-f\left(x_{k}+\lambda_{k} d\right) \geq-\delta \lambda g_{k}^{T} d_{k}$

(c) Approximate Minimization Rule. At each iteration, $\lambda_{k}$ is selected so that

$$
\lambda_{k}=\min \left\{\lambda \mid g\left(x_{k}+\lambda d\right)^{T} d_{k}=0, \lambda>0\right\}
$$

For more details see [13].

In our work we choose Armijo rule to describe the new proposed algorithm in section (5.5).

\section{Review of conjugate gradient method:}

Conjugate gradient (CG) methods were first used to solve the general unconstrained problem by Fletcher and Reeves [6]. Their algorithm (or simple variants) is still frequently used, especially for problems with a large number of variables since they require only a few vectors of length $\mathrm{n}$ to be stored.

Given a symmetric positive definite matrix $G$, the finite set of non-null vectors $\left\{d_{1}, d_{2}, \ldots, d_{k}\right\}$ is said to form a conjugate set if 
$d_{i}^{T} G d_{j}=0$ for all $\mathrm{i} \neq \mathrm{j}$

The CG-method, which we shall now derive, is based on a successive construction of conjugate search directions. Suppose for now that we know the matrix $\mathrm{G}$ defining the quadratic function.

$q(x)=\frac{1}{2}\left(x^{T} G x\right)+b^{T} x \quad ;$

then we can construct a set of conjugate directions $\left\{d_{1}, d_{2}, \ldots, d_{n}\right\}$ from an arbitrary set of linearly independent direction $\left\{r_{1}, r_{2}, \ldots, r_{n}\right\}$ by a Gram Schmidt process in the following way [3] :

Let $d_{1}=r_{1}$, For $\mathrm{i}=1,2, \ldots \ldots \ldots$ n determine successively

$d_{i}=r_{i}+\sum_{j=1}^{i-1} c_{i j} d_{j}$,

where the coefficient $c_{i j}$ must be chosen so that

${ }_{i}^{T} G_{k}=0$ for $\mathrm{k}=1,2, \ldots \ldots, \mathrm{i}-1$

From (13) we get $\quad d_{i}^{T} G d_{k}=r_{i}^{T} G d_{k}+\sum_{j=1}^{i-1} c d_{i j}^{T} G d_{k} \ldots$

By the obvious inductive assumption, substitution of (14) into (15) yields:

$c_{i j}=\left[-r_{i}^{T} G d_{j} / d_{j}^{T} G d_{j}\right]$ For $\mathrm{j}=1,2, \ldots, \mathrm{i}-1$

now $d_{j} \neq 0$, since $r_{j}$ are assumed to be linearly independent, hence $d_{j}^{T} G d_{j}>0$ and $c_{i j}=0$ is well-defined.

Suppose we want to minimize the quadratic function $q$ without first evaluating the Hessian G, but suppose also that we can compute the gradient $\mathrm{g}$ since

$x_{k+1}=x_{k}+\lambda_{k} d_{k}$

$g_{k+1}=G x_{k+1}+b$,

$y_{k}=g_{k+1}-g_{k}=\lambda_{k} G d_{k}$,

$v_{k}=x_{k+1}-x_{k}$,

Let the set $\left\{r_{1}, r_{2}, \ldots, r_{n}\right\}$ be chosen as the set $\left\{d_{1},-g_{2}, \ldots,-g_{n}\right\}$ where $d_{1}$ is an arbitrary downhill direction and $\left\{g_{2}, g_{3}, \ldots, g_{n}\right\}$ are determined successively. Substituting now (17) and using $r_{i}=-g_{i}$ for $\mathrm{i} \geq 2$ into (16) we obtain 
$c_{i j}=\left[g_{i}^{T}\left(g_{j+1}-g_{j}\right) / d_{j}^{T}\left(g_{j+1}-g_{j}\right)\right]=\left[g_{i}^{T} y_{j} / d_{j}^{T} y_{j}\right], \ldots$

for q with an exact line search ( ELS ) we have $d_{i}^{T} g_{j+1}=0$ for $\mathrm{i}=1,2,3, \mathrm{j}$,

also since $\left\{d_{2} d_{3}, \ldots d_{j}\right\}$ were constructed as a linear combination of $\left\{g_{2,} g_{3}, \ldots g_{j}\right\}$ we see that $g_{i}^{T} g_{j}=0$ for $2 \leq \mathrm{i}<\mathrm{j}$

this orthogonally relation makes $c_{i j}=0$ for $\mathrm{j}=2,3, \mathrm{i}-2$

Finally, (13) reduces to the following:

$d_{2}=-g_{2}+\left[g_{2}^{T} y_{1} / d_{1}^{T} y_{1}\right] d_{1}$,

$d_{k+1}=-g_{k+1}+\beta_{k} d_{k}+\gamma_{k} d_{1}$,

$\beta_{k}=\left[g_{k+1}^{T} y_{k} / d_{k}^{T} y_{k}\right]$,

$\gamma_{k}=\left[g_{k+1}^{T} y_{1} / d_{1}^{T} y_{1}\right]$,

This is the Beale CG-method [3] in regular CG-methods the first direction $d_{1}$ is not arbitrary, but it is taken as $-g_{1}$.

Then in this case

$g_{i}^{T} g_{j}=0 \quad$ For $\quad 1 \leq \mathrm{i}<\mathrm{j}$

\subsection{Regular CG-method:}

The regular CG-method can be defined as follows:

given an arbitrary starting point $x_{i}$, define the first search direction by $d_{1}=-g_{1}$ and for $\mathrm{k}=1,2,3, \ldots$ iterate with

$$
\begin{aligned}
& x_{k+1}=x_{k}+\lambda_{k} d_{k}, \\
& \beta_{k}=\left[g_{k+1}^{T} y_{k} / d_{k}^{T} y_{k}\right], \\
& d_{k+1}=-g_{k+1}+\beta_{k} d_{k},
\end{aligned}
$$

the formula (24b) is called the Hestenes and Stiefel [9] form of $\beta_{k}$.It can also be written as: 


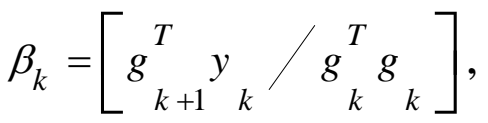

the Polak-Ribiere [11] form ;or

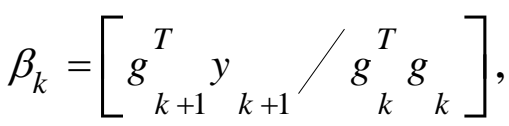

the Fletcher-Reeves [7] form; or

$\beta_{k}=\left[\begin{array}{cccc}-g_{k+1}^{T} & & & \\ & & g^{T}{ }^{T} & \\ & & \\ & & k\end{array}\right]$,

Dixon [5] form.

For the quadratic function in (2) with ELS these four formulae for $\beta_{k}$ are equivalent, but for general function they are not the same and give different algorithms, however, for any arbitrary function $\mathrm{f}$, the CGmethod (24) generates downhill directions provided ELS are used since

$d_{k+1}^{T} g_{k+1}=\left(\begin{array}{ccc}-g_{k+1}+{ }_{k} & d\end{array}\right)^{T} g_{k+1}$,

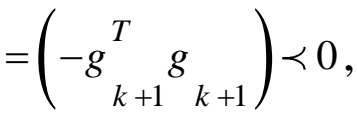

In fact the ELS required can be relaxed since it is clear from (27) that a downhill direction can be obtained whenever

$\beta_{k} d_{k}^{T} g_{k+1}<g_{k+1}^{T} g_{k+1}$,

In practice this is usually replaces by the slightly stronger condition:

$\beta_{k} d_{k}^{T} g_{k+1}<\rho\left(g_{k+1}^{T} g_{k+1}\right)$,

Where $\rho$ is some small constant, say 0.2 or 0.1 (very small positive number) and this condition is attainable in practice for any continuous function. 


\section{Non-Monotone Gradient Methods:}

For the minimization of non-quadratic functions ,non-monotone methods like the gradient method, need to be incorporated with a globalization strategy. The main idea is to accept the step if it is satisfies a weak condition of the form given by (3), when $\mathrm{M}>0$; this condition allows the objective function to increase at some iterations and still guarantees global convergence first in this section we would like to present a general non-monotone gradient algorithm for which we can establish classical convergence results.

\subsection{Non-monotone line search algorithm:}

We adopted a non-monotone line-search strategy, we do not impose decrease of the objective function at every iteration. Instead, we choose a positive integer $\mathrm{M}$ at the beginning of the process and we accept a trial point when a sufficient decrease is obtained in relation to the maximum functional value among the $\mathrm{M}+1$ last iterations.

assume that $\lambda \in(0,1)$ is given independently of the iteration number $\mathrm{k}$ and that $d_{k}$ has been computed .

Step 1 : set $\lambda \longleftarrow 1$.

Step 2 :set $x_{k+1}=x_{k}+\lambda d_{k}$.

Step 3: if $f\left(x_{k}+\lambda_{k} d_{k}\right) \leq \underset{0 \leq \mathrm{j} \leq \min (\mathrm{k}, \mathrm{M})}{\operatorname{Max}} f\left(x_{k-j}\right)+\gamma g_{k}^{T}\left(x_{k+1}-x_{k}\right)$

then define $\lambda_{k}=\lambda$ and finish the line search.

If the condition in step 3 dose not hold, define $\lambda_{\text {new }} \in[0.1 \lambda, 0.9 \lambda]$

Set $\lambda \longleftarrow \lambda_{\text {new }}$ and goto step 2

for more detail see [10].

\subsection{Original Algorithm:}

Outlines of the original non-monotone algorithm [12]:

Step1: Given $x_{0}, \varepsilon=0.05, \mathrm{n}$ is the dimension of the problem , Ac $=1 * \mathrm{E}-4$ $\mathrm{k}=0$, integer $\mathrm{M}>0, \gamma \in(0,1), 0<\delta<1, \alpha=\max (1, \min (1 / \varepsilon,\|g\|)$, $\lambda=1 / \alpha$

Step2: $d_{k}=-g_{k}$

Step 3: If $\left\|g_{k+1}\right\|<A c$ stop else continue.

Step4: $x_{k+1}=x_{k}+\lambda d_{k}$

Step5: Find $\mathrm{z}=\min (\mathrm{k}, \mathrm{M}), 0 \leq j \leq z, \operatorname{Max} f_{(k-j)}-\gamma \cdot \lambda \cdot g_{k}^{T} \cdot g_{k}$

If $f\left(x_{k+1}\right)>\max f(k-j)-\lambda \cdot \gamma \cdot g_{k} \cdot g_{k}^{T}$ choose $\lambda=\delta . \lambda$, $x_{k+1}=x_{k}+\lambda \cdot d_{k}$ 


$$
\text { else } \quad \lambda_{k}=\lambda, \quad x_{k+1}=x_{k}+\lambda_{k} \cdot d_{k} \quad, \quad \alpha=\frac{v^{T} y}{v^{T} v}, \quad \lambda=1 / \alpha
$$

Step 6: $g_{k+1}=\nabla f\left(x_{k+1}\right)$, If $\mathrm{k}=\mathrm{n}$ Consider $\mathrm{k}=1$ and go to (step 2 ), otherwise $\mathrm{k}=\mathrm{k}+1$ and go to (step 4 )

Step7: End

In the next section we are going to draw the flow chart of the original non-monotone algorithm as follows: 


\subsection{Flow chart of the original non-monotone algorithm:}

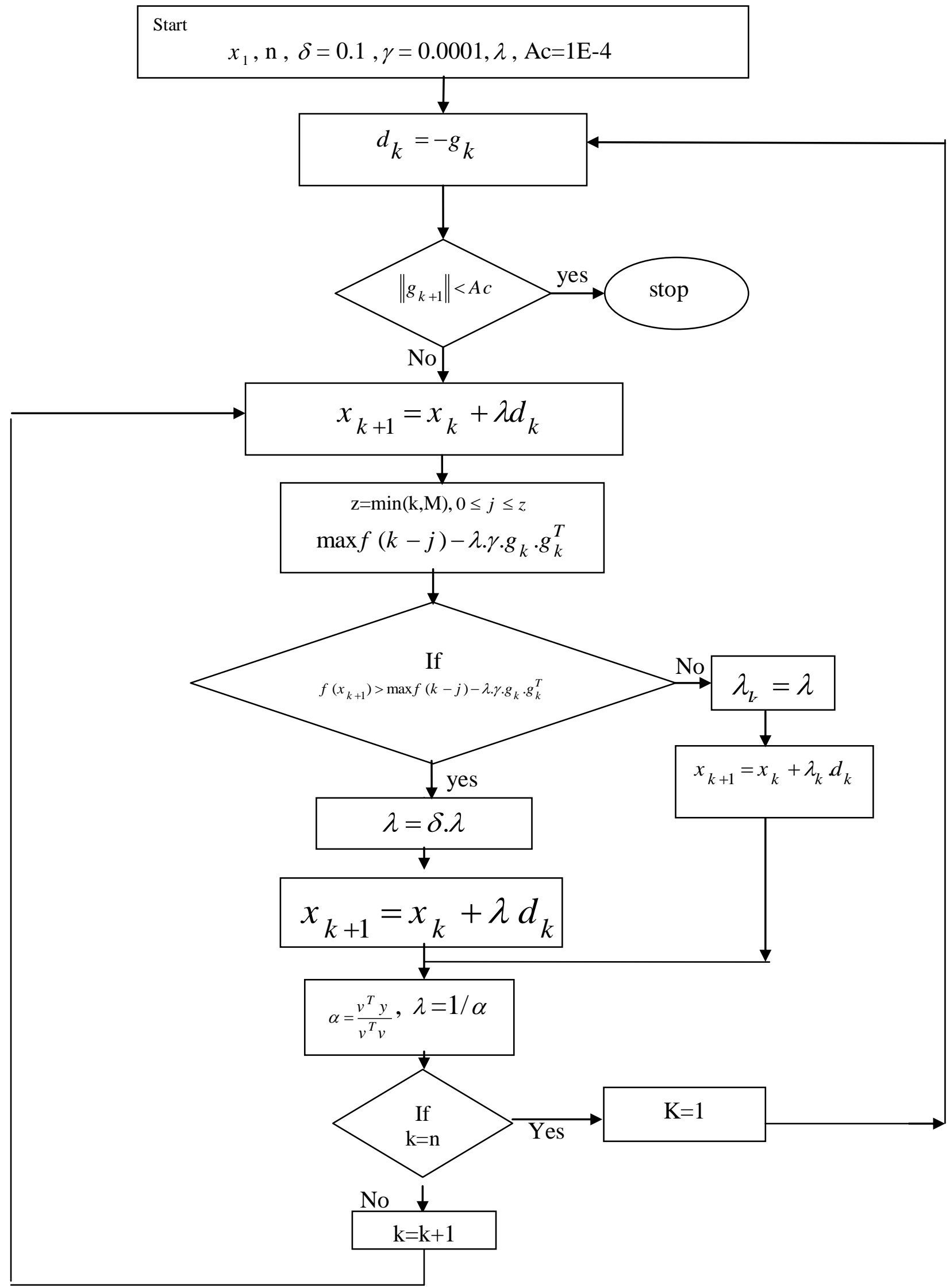




\subsection{Theorem:}

Assume that $\Omega=\left\{\mathrm{x} \in \mathfrak{R}^{n}: f(x) \leq f\left(x_{0}\right)\right\}$ is abounded set. If $f$ is continuously differentiable on an open set that contains $\Omega$ then algorithm 4.2 is well defined and any accumulation point of the sequence $\left\{x_{k}\right\}$ that generates stationary point.

For the details of the proof see [12].

\section{Variable Metric (VM) methods:}

Quasi-Newton methods are probably the most popular general purpose algorithm for unconstrained optimization problems .Many QN-method are advantageous due to their fast convergence and absence of second order derivatives computation.

For the QN-methods assume that at the $\mathrm{k}$ - th iteration at approximation point $\mathrm{x}$ and $\mathrm{n} \times \mathrm{n}$ matrix $H_{k}$ are available, then the methods proceed by generating a sequence of approximation points via the equation:

$x_{k+1}=x_{k}+\lambda_{k} d_{k} \quad, d_{k}=-H_{k} g_{k}$

where $H_{k}$ is an approximation of $G_{k}^{-1}$ which is corrected for updating from iteration to iteration .In general $H_{k}$ is symmetric and positive definite ,there are different choices of $H_{k}$ see [7] we list here some most popular forms

$$
H_{k+1}^{S R 1}=H_{k}+\frac{\left(v_{k}-H_{k} y_{k}\right)\left(v_{k}-H_{k} y_{k}\right) T}{\left(v_{k}-H_{k} y_{k}\right)^{T} y_{k}},
$$

is called rank one correction formulae, where

$v_{k}=x_{k+1}-x_{k} \quad$ and $\quad y_{k}=g_{k+1}-g_{k}$

$$
H_{k+1}^{D F P}=H_{k}+\frac{v_{k} v_{k}^{T}}{v_{k}^{T} y_{k}}-\frac{H_{k} y_{k} y_{k}{ }^{T} H_{k}}{y_{k}^{T} H_{k} y_{k}},
$$

is the DFP formula

$$
H_{k+1}^{B F G S}=H_{k}+\left[1+\frac{y_{k}^{T} H_{k} y_{k}}{v_{k}^{T} y_{k}}\right] \frac{v_{k} v_{k}^{T}}{v_{k}^{T} y_{k}}-\left[\frac{v_{k} y_{k}^{T} H_{k}+H_{k} y_{k} v_{k}^{T}}{v_{k}^{T} y_{k}}\right] ;
$$

all three forms satisfy the quasi-Newton condition $H_{k+1} y_{k}=v_{k}$ and maintain positive definite matrices if $H_{1}$ is positive definite. And we use the last one (BFGS) to update $H_{k}$ in our new proposed algorithm and the scalar of HS to scale the direction $d_{k}$.

\subsection{Preconditioned CG algorithm (PCG):}

The preconditioned CG methods (PCG) first appeared in paper by Axelsson in 1972 , it was developed with object of accelerating the convergence of the CG-method by a transformation of variables while keeping the basic properties of the method. Such transformation was 
introduced by Allwright in 1972, the symmetric positive definite matrix $H$ can be factored in various ways for example as $H=L L^{T}$ where $L$ is lower triangular and non-singular.

Buckly in 1978 [4] introduced an algorithm in which conjugate gradient and quasi-Newton search directions occur together and which can be extended this type of algorithms see for example [1].

The search direction to the preconditioned (PCG) method defined by:

$d_{t}=-H_{k} g_{t}$

$d_{k+1}=-H_{k} g_{k}+\beta_{k} d_{k}$ for $k \geq 1$

$\beta_{H S}=\frac{g_{k+1}^{T} H y_{k}}{d_{k}^{T} H y_{k}}$

where $H$ is the BFGS update defined as :

$H_{i+1}^{B F G S}=H_{i}-\left[\frac{H_{i} y_{t} v_{t}^{T}}{v_{t}^{T} y_{t}}\right]+v_{t}\left[\frac{v_{t}^{T} y_{t}+y_{t}^{T} H_{i} y_{t}}{\left(v_{t}^{T} y_{t}\right)^{2}}-\frac{H_{i} y_{t}}{v_{t}^{T} y_{t}}\right]^{T}$

For more detail we state the following algorithm (Buckely,1978):

Algorithm (Buckley, 1978)

Start with $x_{1}, H_{1}=I$, let $\mathrm{t}=1, \mathrm{i}=1, \varepsilon>0$.

Step 1:let $d_{t}=-H_{i} g_{t}$ if $\left\|g_{t}\right\|<\varepsilon$

Step2: $\forall \mathrm{k}=\mathrm{t}, \mathrm{t}+1, \mathrm{t}+2, \ldots$ repeat with $x_{k+1}=x_{k}+\lambda_{k} d_{k}$

$$
d_{k+1}=-H_{i} g_{k+1}+\frac{y_{k}^{T} H_{i} y_{k}}{d_{k}{ }^{T} y_{k}} d_{k} \text { where } y_{k}=g_{k+1}-g_{k}
$$

Step 3: we use the restarting when $\left|\frac{g_{k}^{T} H_{i} g_{k+1}}{g_{k+1}^{T} H_{i} g_{k+1}}\right|>0.2$

when this condition is satisfied it restarts $\mathrm{t}$ to $\mathrm{k}$ and go to step 4

Step4: Update $H_{i}$ by $H_{i+1}$ (using BFGS formula) to get:

$$
H_{i+1}^{B F G S}=H_{i}-\left[\frac{H_{i} y_{t} v_{t}^{T}}{v_{t}^{T} y_{t}}\right]+v_{t}\left[\frac{v_{t}^{T} y_{t}+y_{t}^{T} H_{i} y_{t}}{\left(v_{t}^{T} y_{t}\right)^{2}}-\frac{H_{i} y_{t}}{v_{t}^{T} y_{t}}\right]^{T}
$$

Step 5: Replace $\mathrm{i}$ with $\mathrm{i}+1$ and go to step1.

\subsection{Self-Scaling PCG method:}

Clearly self-scaling techniques are very effective in unconstrained optimization algorithms. In this section a new PCG method for solving unconstrained optimization problems is proposed. 


\section{ABBAS Y. AL-BAYATI\&ASEEL MOAYAD}

This PCG algorithm considered here has an additional property of being invariant under scaling of the function is twice continuously differentiable and search direction is descent i.e $g_{k}^{T} d_{k}<0$ also we assume that line search is exact i.e $g_{k}^{T} d_{k}=0$

Let $d_{k+1}=-H_{k} g_{k+1}+\beta_{k} d_{k}$

where $\beta_{k}$ as in (33) where the matrix $H_{k}$ is an approximation of $G^{-1}$ the inverse of Hessian of the objective function $f(\mathrm{x})$. One important feature of PCG method is the choice of $H_{k}$ the method requires $H_{k}$ to be positive definite to deduce directions.

\subsection{Theorem (New proof for the positive definite $H_{k}$ in equation} (34)):

Let $G$ be a $\mathrm{n} \times \mathrm{n}$ symmetric matrix , let $\mathrm{G} v=\mathrm{y}$ where $\mathrm{y}_{\mathrm{k}}=\mathrm{g}_{\mathrm{k}+1}-\mathrm{g}_{\mathrm{k}}$ and $v_{k}=x_{k+1}-x_{k}$ and let $m_{0}=v_{k}^{T} v_{k}, \mathrm{~m}_{1}=\mathrm{y}_{\mathrm{k}}^{\mathrm{T}} \mathrm{v}_{\mathrm{k}}, m_{2}=y_{k}^{T} y_{k}$ then the quantity

$$
\alpha=\frac{\mathrm{m}_{1}}{\mathrm{~m}_{0}}
$$

is an approximation for an eigen value $\lambda$ of $G$ and if we set $\alpha=\lambda+\varepsilon$, so that $\varepsilon$ is the error of $\alpha$, then $|\varepsilon| \leq \sqrt{\frac{\mathrm{m}_{2}}{\mathrm{~m}_{0}}-\alpha^{2}}$,

Proof:

Let $\delta^{2}$ denote the radiant in (36) then since $\mathrm{m}_{1}=\alpha \mathrm{m}_{0}$, we have $(y-\alpha v)^{\mathrm{T}}(\mathrm{y}-\alpha \mathrm{v})=\mathrm{m}_{2}-2 \alpha \mathrm{m}_{1}+\alpha^{2} \mathrm{~m}_{0}=\mathrm{m}_{2}-\alpha^{2} \mathrm{~m}_{0}=\delta^{2} \mathrm{~m}_{0}$ ,............(37)

Since $G$ is symmetric, it has an orthogonal set of $\mathrm{n}$ real unit eigen vectors $z_{1}, z_{2}, \ldots \ldots \ldots \ldots \ldots . . . z_{n}$ corresponding to the eigen values $\lambda_{1}, \ldots \ldots \ldots \ldots, \lambda_{\mathrm{n}}$

respectively. (some of them may be equal).Then $\mathrm{v}$ has a representation of the form

$$
\mathrm{v}=\mathrm{a}_{1} \mathrm{z}_{1}+\ldots+\mathrm{a}_{\mathrm{n}} \mathrm{z}_{\mathrm{n}}
$$

now $\mathrm{Gz}_{1}=\lambda_{1} \mathrm{z}_{1}$, etc , and we obtain $\mathrm{y}=\mathrm{Gv}=\mathrm{a}_{1} \lambda_{1} \mathrm{z}_{1}+\ldots+\mathrm{a}_{\mathrm{n}} \lambda_{\mathrm{n}} \mathrm{z}_{\mathrm{n}}$ and , since the $z_{i}$ are orthogonal unit vectors

, $\mathrm{m}_{0}=\mathrm{v}^{\mathrm{T}} \mathrm{v}=\mathrm{a}_{1}^{2}+\ldots+\mathrm{a}_{\mathrm{n}}^{2}$

it follows that (37)

$\mathrm{y}-\alpha \mathrm{v}=\mathrm{a}_{1}\left(\lambda_{1}-\alpha\right) \mathrm{z}_{1}+\ldots+\mathrm{a}_{\mathrm{n}}\left(\lambda_{\mathrm{n}}-\alpha\right) \mathrm{z}_{\mathrm{n}}$ since the $z_{i}$ are orthogonal unit vectors we thus obtain from (37) 
$\delta^{2} \mathrm{~m}_{0}=\mathrm{a}_{1}^{2}\left(\lambda_{1}-\alpha\right)^{2}+\ldots+\mathrm{a}_{\mathrm{n}}^{2}\left(\lambda_{\mathrm{n}}-\alpha\right)^{2}$

replacing each $\left(\lambda_{i}-\alpha\right)^{2}$ by the smallest of these terms, we have from (39) $\delta^{2} \mathrm{~m}_{0} \geq\left(\lambda_{\mathrm{c}}-\alpha\right)^{2}\left(\mathrm{a}_{1}^{2}+\ldots+\mathrm{a}_{\mathrm{n}}^{2}\right)=\left(\lambda_{\mathrm{c}}-\alpha\right)^{2} \mathrm{~m}_{0}$

Where $\lambda_{\mathrm{c}}$ is an eigen value to which $\alpha$ is closest dividing this inequality by

$\mathrm{m}_{0}$ and taking square root, we obtain (36). And the theorem is proved.

Because the matrix $G$ is positive definite then all eigen values are positive.

\subsection{Theorem (New descent property):}

The new search direction which is defined in

$d_{k+1}=-g_{k+1}+\frac{v_{k}^{T} v_{k}}{v_{k}^{T} y_{k}} \frac{g_{k+1} y_{k}^{T}}{d_{k}^{T} y_{k}} d_{k}$,

is always negative with exact line search.

Proof:

Multiplying (40) by $g_{k+1}$ then we have

$$
\begin{aligned}
& d_{k+1}^{T} \cdot g_{k+1}=-g_{k+1}^{T} \cdot g_{k+1}+\frac{v_{k}^{T} v_{k}}{v_{k}^{T} y_{k}} \frac{g_{k+1} y_{k}^{T}}{d_{k}^{T} y_{k}} d_{k} g_{k+1}^{T} \\
& =-g_{k+1}^{T} g_{k+1}+\frac{v_{k}^{T} v_{k}}{v_{k}^{T} y_{k}} \frac{g_{k+1}^{T} g_{k+1}}{d_{k}^{T} y_{k}} d_{k} y_{k}^{T}=-g_{k+1}^{T} g_{k+1}\left(1-\frac{v_{k}^{T} v_{k}}{v_{k}^{T} y_{k}}\right)
\end{aligned}
$$

Because $\frac{v_{k}^{T} v_{k}}{v_{k}^{T} y_{k}}$ is a $n \times n$ approximation for an eigen value of $H$, then $\frac{v_{k}^{T} v_{k}}{v_{k}^{T} y_{k}}>0$ and always we have the direction $d_{k+1}$ is negative.

\subsection{New PCG- algorithm with dynamical retards:}

Out lines of the new proposed PCG algorithm:

Let kret : number of retard .

$\mathrm{knm}$ : number of non-monotone .

Step1: Given $x_{1}, \mathrm{n}$ is the dimension of the problem, $\mathrm{k}=1, \mathrm{Ac}=1 \times 10^{-4}$ integer $\mathrm{M}>0,0<\delta<1, H_{0}=I, \gamma=0.001, \mathrm{knm}=0$, kret $=0$

Step 2: $\beta=1, d_{k}=-H_{k} g_{k}, z=f\left(x_{k}\right)$

Step 3: $A r=1$

Step 4: If $(A r>1)$ go to step 5(a) else $\lambda=\beta$ go to step 6

Step 5 (a): If (Ar > 2) go to step 5(b) else $\beta=0.2, \lambda=\beta \quad$ go to step 6

Step 5(b) : $\lambda=\beta^{A r-1}$ 
Step 6: $\quad x_{k+1}=x_{k}+\lambda_{k} d_{k}$, compute $f_{k+1}=f\left(x_{k+1}\right)$

Step 7: If $f_{k+1}-z \leq 0.1 \cdot \lambda\left(g_{k}{ }^{T} d_{k}-0.5 \cdot \lambda \cdot g_{k}{ }^{T} d_{k}\right)$ go to step 8

else $\quad A r=A r+1$ go to step 4

Step 8: for $\mathrm{j}=0$, $\min (\mathrm{k}, \mathrm{M})$ find $\max f_{(k-j)}-\gamma \cdot \lambda \cdot g_{k}^{T} \cdot g_{k}$

Step 9: if $f_{k+1}>\max f_{(k-j)}-\gamma \cdot \lambda \cdot g_{k}^{T} \cdot g_{k}$ then $\lambda=\delta \cdot \lambda, \mathrm{knm}=\mathrm{knm}+1$

Step 10: $N O I=N O I+1$

$$
x_{k+1}=x_{k}+\lambda . d_{k} \quad \text { else } \quad \text { kret }=\mathrm{kret}+1 \quad x_{k+1}=x_{k}+\lambda \cdot d_{k}
$$

Step 11: If $\left\|g_{k+1}\right\|<A C$ then go to step 15 else continue.

Step 12: $\alpha=\frac{v_{k}^{T} y_{k}}{v_{k}^{T} v_{k}}$ Compute $H_{k+1}$ by the BFGS update and compute the

$$
\text { scalar } \beta_{k}^{H S} \text { where } \quad \beta_{k}^{H S}=\frac{g_{k+1}{ }^{T} H y_{k}}{d_{k}^{T} H y_{k}}
$$

Step 13:Compute a new direction $d_{k+1}=-H_{k} g_{k+1}+1 / \alpha \beta_{k}^{H S} d_{k}$

Step 14: If $\left(g_{k+1}^{T} d_{k+1}>0\right.$ or $\left.\mathrm{k}=\mathrm{n}\right) \quad$ then $\mathrm{k}=1$ and go to step 2 else $\mathrm{k}=\mathrm{k}+1$ go

$$
\text { to step } 3
$$

Step 15: End

And now we are going to represent the flowchart of the new proposed PCG- algorithm as follows: 
A new self scaling PCG algorithm

5.6 Flow chart for the new proposed algorithm:

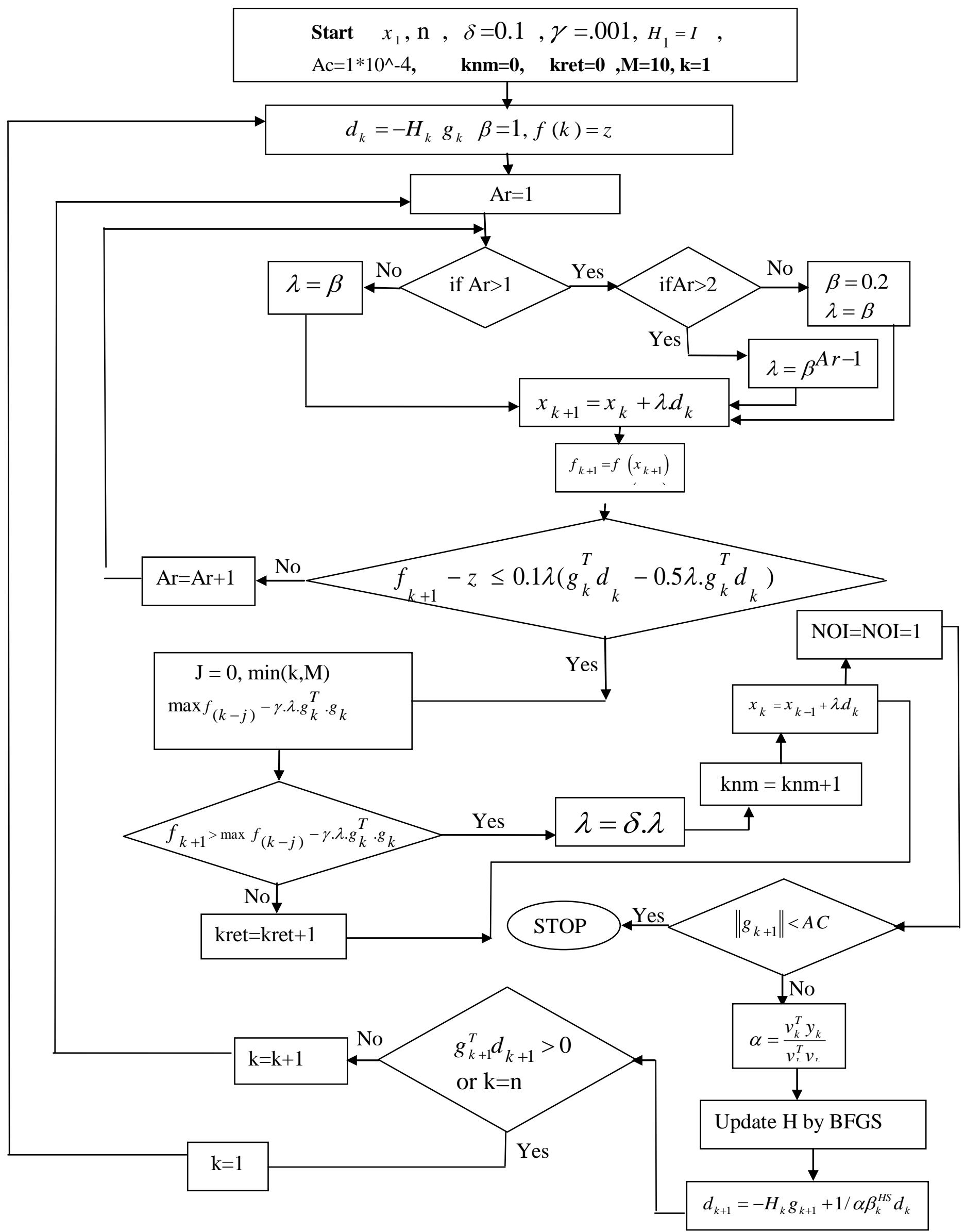




\subsection{The Global Convergence Theorem (for the new proposed algorithm):}

If $f\left(x_{k}\right) \leq f_{k}^{i} \leq \operatorname{Max}_{0 \leq \mathrm{j} \leq \min (\mathrm{k}, \mathrm{M})} f\left(x_{k-j}\right)+\gamma g_{k}^{T}\left(x_{k+1}-x_{k}\right)$

and $\quad f^{i}\left(x_{k}+\lambda_{k} d_{k}\right)=f\left(X_{k}\right)$

holds, then the algorithm is well defined in the sense that

$f\left(x_{k}+\lambda_{k} d_{k}\right) \leq \underset{0 \leq \mathrm{j} \leq \min (\mathrm{k}, \mathrm{M})}{\operatorname{Max}} f\left(x_{k-j}\right)+\gamma g_{k}^{T}\left(x_{k+1}-x_{k}\right)$

hold for finite (M).

Proof:

Assume by contradiction that, at the iteration $\mathrm{k}$, the test (43) is never satisfied then there exist sequence $\left\{\lambda_{M}\right\}$, with $\lambda_{M} \longrightarrow 0$ as $M \longrightarrow \infty$ such that

$$
f\left(x_{k}+\lambda_{k} d_{k}\right) \geq \max _{0 \leq j \leq \min (k, M)} f\left(x_{k-j}\right)+\gamma g_{k}^{T}\left(x_{k+1}-x_{k}\right),
$$

Remember now that, because of (41) we have that $f^{i}{ }_{k}-f\left(x_{k}\right) \geq 0$ now if $f^{i}{ }_{k}-f\left(x_{k}\right)>0$ since $\lambda_{M} \rightarrow 0$ for sufficiently large $M,(44)$ yields that $f^{i}{ }_{k}-f\left(x_{k}\right) \leq 0$, which is a contradiction, if instead $f^{i}{ }_{k}-f\left(x_{k}\right)=0$, then dividing both term of (44) by $\lambda_{M}$ and taking the limit for $M \longrightarrow 0$ we obtain that $g_{k}^{T} d_{k} \geq \gamma g_{k}^{T} d_{k}$.

\section{Numerical results and conclusions:}

\section{1 numerical results:}

The two proposed algorithms describes in this paper namely:

1. The original non monotone algorithm (original)

2. The new proposed algorithm (new)

They are programmed in double precision (FORTRAN 95). The complete set of results are given in Tables (6.1.1) - (6.1.4). The numerical comparison are the number of function evaluations NOF, number of iterations NOI; number of non monotones (NONM) and the number of dynamical retards (NODR) are considered. The actual convergence criterion employed was $\left\|g_{k+1}\right\|<1 * 10^{\wedge}-4$ for all the algorithms twenty well-known test functions with different dimensions are employed in this comparison. 
The first table contains the results of ten test functions for $n=4$ only, while the second table contains another ten test function of dimensionality one hundred and one thousand.

Table (6.1.1)

Comparison between the original \& new algorithm

for $\mathrm{n}=4$ only

(standard test functions)

\begin{tabular}{|c|c|c|c|c|c|c|c|c|}
\hline \multirow{2}{*}{} & \multicolumn{4}{|c|}{ Origin } & \multicolumn{4}{c|}{ New } \\
\cline { 2 - 10 } & NOI & NOF & NONM & NODR & NOI & NOF & NONM & NODR \\
\hline ROSEN & 96 & 193 & 25 & 96 & 61 & 146 & 12 & 49 \\
\hline BEALE & 40 & 81 & 8 & 40 & 13 & 34 & 0 & 11 \\
\hline DIXON & 39 & 79 & 10 & 39 & 13 & 32 & 0 & 13 \\
\hline WOLFE & 23 & 47 & 4 & 23 & 10 & 30 & 0 & 10 \\
\hline CANTRAL & 59 & 119 & 12 & 59 & 37 & 82 & 0 & 37 \\
\hline SUM & 14 & 29 & 0 & 14 & 16 & 36 & 0 & 16 \\
\hline WOOD & 333 & 667 & 83 & 333 & 45 & 118 & 5 & 40 \\
\hline MILEL & 181 & 363 & 49 & 181 & 28 & 62 & 0 & 28 \\
\hline CUBIC & 159 & 319 & 42 & 159 & 76 & 184 & 19 & 57 \\
\hline POWELL & 282 & 565 & 77 & 282 & 32 & 76 & 0 & 32 \\
\hline \hline TOTAL & 1226 & 2462 & 310 & 1226 & 331 & 800 & 36 & 293 \\
\hline
\end{tabular}

From the above table it is clear that the new proposed algorithms has an improvements on the original non monotone algorithm (origin) by taking $100 \%$ [ $\mathrm{NOI} ; \mathrm{NOF}$ ] for the origin algorithm we have in table (6.1.2)

Table (6.1.2)

Performance percentage of the new algorithm compared with the original algorithm

\begin{tabular}{|c|c|c|}
\hline Tools & NOI & NOF \\
\hline Origin & $100 \%$ & $100 \%$ \\
\hline new & $26 \%$ & $32 \%$ \\
\hline
\end{tabular}

It is clear from the above table, for small dimensionality test function $(\mathrm{n}=$ 4)

and for ten selected test functions there are an important of $74 \%$ NOI ; $68 \%$ NOF . 


\section{ABBAS Y. AL-BAYATI\&ASEEL MOAYAD}

Table (6.1.3)

Comparison between the (origin) and (new) algorithms for dimensionality $\mathrm{n}=100$ and $\mathrm{n}=1000$

\begin{tabular}{|c|c|c|c|c|c|c|c|c|c|}
\hline & \multirow[b]{2}{*}{$\mathbf{N}$} & \multicolumn{4}{|c|}{ Origin } & \multicolumn{4}{|c|}{ New } \\
\hline & & NOI & NOF & $\begin{array}{c}\text { NON } \\
\text { M }\end{array}$ & $\begin{array}{c}\text { NOD } \\
\mathbf{R} \\
\end{array}$ & NOI & NOF & $\begin{array}{c}\text { NON } \\
\text { M }\end{array}$ & $\begin{array}{c}\text { NOD } \\
\text { R }\end{array}$ \\
\hline MILEL & 100 & 105 & 211 & 0 & 105 & 28 & 67 & 0 & 28 \\
\hline BEALE & 100 & 49 & 99 & 0 & 99 & 19 & 53 & 0 & 16 \\
\hline DIXON & 100 & 1492 & 2985 & 322 & 1492 & 160 & 495 & 114 & 46 \\
\hline WOLFE & 100 & 44 & 89 & 2 & 44 & 110 & 422 & 66 & 44 \\
\hline $\begin{array}{c}\text { CANTR } \\
\text { AL }\end{array}$ & 100 & 94 & 189 & 0 & 94 & 40 & 87 & 0 & 40 \\
\hline MILEL & $\begin{array}{c}100 \\
0\end{array}$ & 130 & 261 & 0 & 130 & 40 & 100 & 0 & 40 \\
\hline BEALE & $\begin{array}{c}100 \\
0\end{array}$ & 49 & 99 & 0 & 49 & 28 & 73 & 0 & 19 \\
\hline Shallo & $\begin{array}{c}100 \\
0\end{array}$ & 23 & 47 & 0 & 23 & 19 & 48 & 0 & 19 \\
\hline $\begin{array}{c}\text { CANTR } \\
\text { AL }\end{array}$ & $\begin{array}{c}100 \\
0\end{array}$ & 111 & 223 & 0 & 111 & 42 & 91 & 0 & 42 \\
\hline $\begin{array}{c}\text { POWEL } \\
\text { L }\end{array}$ & $\begin{array}{c}100 \\
0\end{array}$ & 120 & 241 & 1 & 120 & 397 & 1426 & 91 & 306 \\
\hline TOT & & 2217 & 4444 & 325 & 2267 & 883 & 2862 & 271 & 600 \\
\hline
\end{tabular}

From the above table it is clear that the new proposed algorithms has an improvements on the original Non-monotone algorithm (origin)by taking $100 \%$ [NOI ; NOF] for the origin algorithm we have in table (6.1.4) 
Table (6.1.4)

Performance percentage of the new algorithm compared with the original algorithm

\begin{tabular}{|c|c|c|}
\hline Tools & NOI & NOF \\
\hline Origin & $100 \%$ & $100 \%$ \\
\hline new & $39 \%$ & $64 \%$ \\
\hline
\end{tabular}

It is clear from the above table, for the dimensionality test function $100 \leq \mathrm{n} \leq 1000$ there are an improvements of $61 \% \mathrm{NOI} ; 36 \% \mathrm{NOF}$.

\subsection{Conclusions:}

In this paper a new PCG-algorithm which employs a Non-monotone search direction with dynamical retards is investigated both theoretically and numerically.

The new proposed algorithm employs a modified line search sub program and it saves about 77\% of NODR (Dynamical Retards) for small size of test functions while it saves a bout $74 \%$ of NODR for high-size test problems.

\section{Appendix:}

All the test function used in this paper is from general literature [2]:

1. Cube function,

$$
F(x)=100\left(x_{2}-x_{1}^{3}\right)^{2}+\left(1-x_{1}\right)^{2}, \quad x_{0}=(-1.2,1.0)^{T}
$$

2. Beale function,

$$
\begin{aligned}
& F(x)=\left(1.5-x_{1}\left(1-x_{2}\right)\right)^{2}+\left(2.25-x_{1}\left(1-x_{2}^{2}\right)\right)^{2}+\left(2.625-x_{1}\left(1-x_{2}^{3}\right)\right)^{2}, \\
& x_{0}=(0,0)^{T}
\end{aligned}
$$

3. Miele and Cornwell function,

$$
\begin{aligned}
& F(x)=\left(e^{x_{1}}-1\right)^{2}+\tan ^{4}\left(x_{3}-x_{4}\right)+100\left(x_{2}-x_{3}\right)^{6}+x_{1}{ }^{8}+\left(x_{4}-1\right)^{2}, \\
& x_{0}=(1,2,2,2)^{T}
\end{aligned}
$$


4. Dixon function,

$$
F(x)=\left(1-x_{1}\right)^{2}+\left(1-x_{10}\right)^{2}+\sum_{i=2}^{n}\left(x_{i}^{2}-x_{i+1}\right)^{2}, \quad x_{0}=(-1 ; \ldots)^{T}
$$

5. Shallow function,

$$
F(x)=\sum_{i=1}^{n / 2}\left[\left(x_{2 i-1}^{2}-x_{2 i}\right)^{2}+\left(1-x_{2 i-1}\right)^{2}\right] \quad x_{0}=(-2 ; \ldots)^{T}
$$

6. Powell function (generalized form),

$$
\begin{aligned}
& \left.F(x)=\sum_{i=1}^{n / 4}\left[\left(x_{4 i-3}\right)+10 x_{4 i-2}\right)^{2}+5\left(x_{4 i-1}-x_{4 i}\right)^{2}+\left(x_{4 i-2}-2 x_{4 i-1}\right)^{4}+10\left(x_{4 i-3}-x_{4 i}\right)^{4}\right] \\
& x_{0}=(3,-1,0,1 ; \ldots)^{T}
\end{aligned}
$$

7. Wood function (generalized form),

$$
\begin{aligned}
& F(x)=\sum_{i=1}^{n / 4}\left[100\left(x_{4 i-2}-x_{4 i-3}^{2}\right)^{2}+\left(1-x_{4 i-3}\right)^{2}+90\left(x_{4 i}-x_{4 i-1}^{2}\right)^{4}+\left(1-x_{4 i-1}\right)^{2}+\right. \\
& \left.10.1\left(x_{4 i-2}-1\right)^{2}+\left(x_{4 i}-1\right)^{2}+19.8\left(x_{4 i-2}-1\right)\left(x_{4 i}-1\right)\right], x_{0}=(-3,-1,-3,-1 ; \ldots)^{T}
\end{aligned}
$$

8. Rosenbrock Banana function,

$$
\mathrm{F}(\mathrm{x})=100\left(x_{2}-x_{1}^{2}\right)^{2}+\left(1-x_{1}\right)^{2}, \quad x_{0}=(-1.2,1.0)^{T}
$$

9. Wolfe function,

$$
\begin{aligned}
& F(x)=\left(-x_{1}\left(3-x_{1} / 2\right)+2 x_{2}-1\right)^{2}+\sum_{i=1}^{n-1}\left(x_{i-1}-x_{i}\left(3-x_{i} / 2\right)+2 x_{i+1}-1\right)^{2} \\
& +\left(x_{n-1}-x_{n}\left(3-x_{n} / 2\right)-1\right)^{2}, x_{0}=(-1 ; \ldots)^{T}
\end{aligned}
$$

10. Sum function,

$\mathrm{F}(\mathrm{x})=\left(x_{1}-1\right)^{4}, \quad x_{0}=(2 ; \ldots)^{T}$

11.Cantral function

$\mathrm{F}(\mathrm{x})=$

$\sum_{i=1}^{n / 4}\left[\left(\exp \left(x_{4 i-3}\right)-x_{4 i-2}\right)^{4}+100\left(x_{4 i-1}-x_{4 i-1}\right)^{6}+\left(\arctan \left(x_{4 i-1}-x_{4 i}\right)\right)^{4}+x_{4 i-3}\right]$
$x_{0}=(1,2,2,2)^{T}$ 


\section{References :}

1.AL-Bayati A.Y, ( 1996) "Anew PCG method for unconstrained Nonlinear optimization", Iraq,vol(5),No.1,pp.71-92.

2.AL-Bayati A.Y , (2001),"New Generalized CG-methods for the Nonquadratic Model In unconstrained optimization" ,Journal of AL-yarmok , Jordan ,vol (10), pp 1-17.

3. Beal,E.M.L. (1972)."A Derivation of Conjugate Gradient". In: Numerical Methods For Nonlinear Optimization. Edited by Lootsma, F.A., Academic Press, pp.39-43.

4. Buckley, A. G. (1978). "A Combined Conjugate Gradient QuasiNewton Minimization Algorithm" . Math.Prog.Vol.15, pp.200-210.

5. Dixon L.C.W ; spedicato E. and Szego G.P. (1980), "Non Linear Optimization Theory and Algorithms", USA.

6.Fletcher, R. and reeves.C.M.(1964)."Function Minimization by Conjugate Gradients". Computer Journal, 7, pp.149-54.

7. Fletcher, R. (1987). "Practical Method of Optimization". John Wiley and Sons, Chichester, New York, ,Britain, Toronto , and Singapore.

8.Grippo, F. Lampariello, and S.LUCID, (1986), "Anonmonotone line search technique for Newton's method", SIAM J. Number. Anal., 23, pp.707-716.

9. Hestense ,M.R. and Stiefel, E. (1952). "Methods of Conjugate Gradient for solving Linear System". Journal Res .N.B.S., Vol.49, pp.449-464.

10.Martinez ,J.M. ,Pilotta,E.A., Raydan,M., (2003),"Spectral Gradient Methods for Linearly Constrained", special communication.

11. polak ,E. and Ribier G.,(1969), "Note Sur La Convergence Des Methods De Direction Conjugate ", Rev. Frinfr, Rech Oper.,16.R1.

12. Raydan ,F., (2003)"Gradient Method With Dynamical Retards For Large-Scale Optimization Problems", Kent state university,vol.16,pp.186193.

13. Zhen-jun shi. and Jie shen,( 2005) "step-size Estimation For Unconstrained Optimization Methods" university of Michigan ,Dearborn , MI 48128, USA, vol(24), N.3, pp.399-416. 